\title{
How sleep may be key to antisocial behaviour in young people
}

Sleep is vital to our health and yet remains understudied in young people and especially in its links to behaviours. is focused on highlighting the role of sleep in problem behaviours in adolescents. His work on sleep unearths some important influences on deviant behaviour but also points to ways that young people can be supported to avoid such Professor Alexander Vazsonyi

s anyone with a newborn will tell linked to circadian rhythms - our internal you, sleep is vital to our ability body clock - dictating our sleepiness at to function at our best. Research over the last years has shown that sleep is key to healnyliving and a crucial health. Sleep quality and length has been linked to a number of health issues including heart and blood vessel health, cognitive conditions, obesity and cancer risk. The quality of sleep has also been associated with depression and anxiety. with poor sleep involved in almost all. psychiatric conditions.

Sleep need changes over a lifetime, with newborns sleeping for the majority of the day, teenagers needing up to ten hours and mid-life adults requing around eight hours of sleep. Sleep is intrinsically

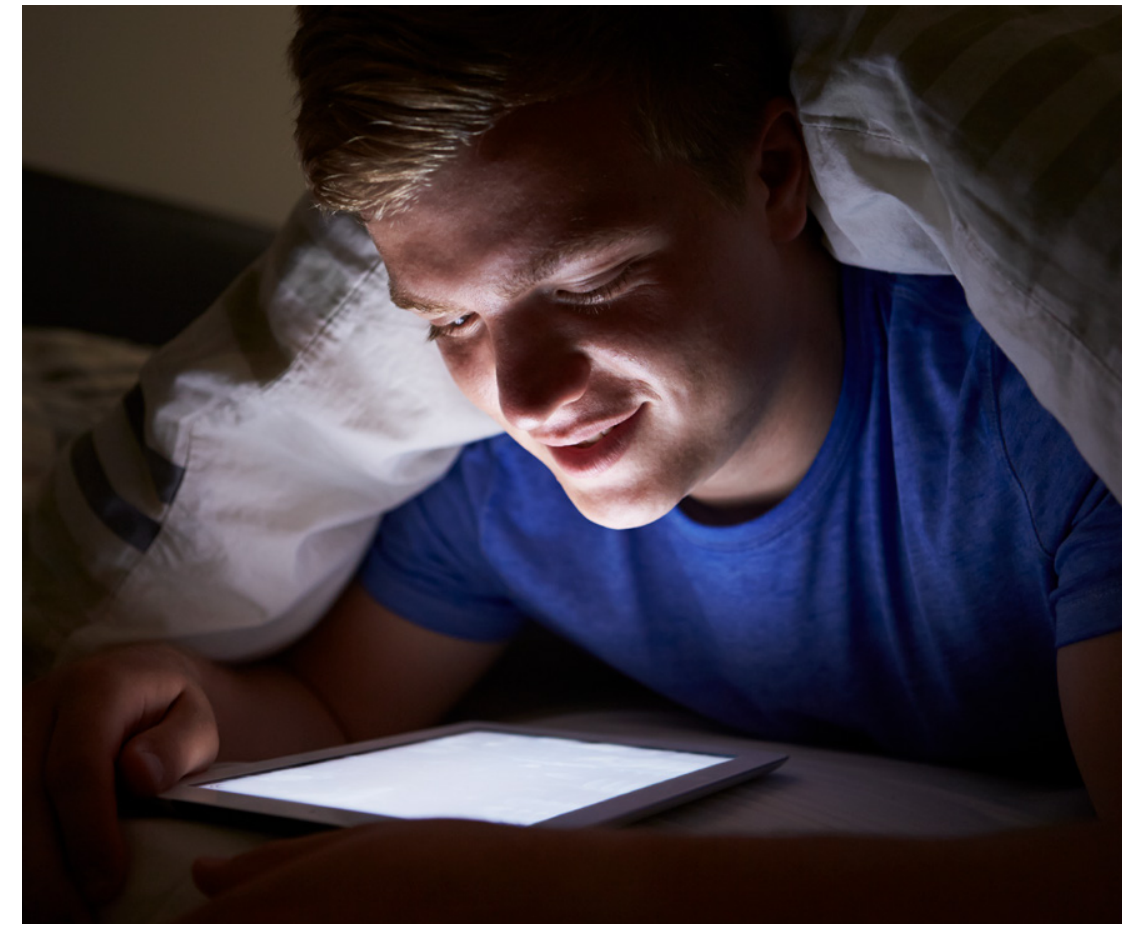

certain times of day. Adolescents typically have an 'Owl' chronotype, staying up The natura sleep cycle for teens ofter. conflicts with their school scheduth, and this period of life is notoriously linked with poor sleep.

Signs of poor sleep include trouble falling asleep, waking up multiple times throughout the night, excessive daytim sleepiness, and not being able to fall back asleep after waking. Poor sleep makes it difficult to concentrate and to store and retain information, and lack of sleep can impair school performance for many adolescents. Psychologists are also starting to understand that poor turning their attention to how sle are urning their attention to how sleep

\section{SLEEP AND BEHAVIOUR}

Sleep is known to be related to 'normviolating' or deviant behaviours - actions that go against the informal rules of how society works. Precisely how sleep affects these forms of behaviours Professor of Family Sciences and Director of the Adolescent Development Lab at the University of Kentucky, is an work focuses on the transition between childhood and adolescence and the onset of problem behaviours, violence and deviance. Prof Vazsonyi - who is altor-In-Chief of the Journal of Early Adolescence - is interested in the role of culture and wider societal seep can be linked to aggression and authority in the field of adolescence. His

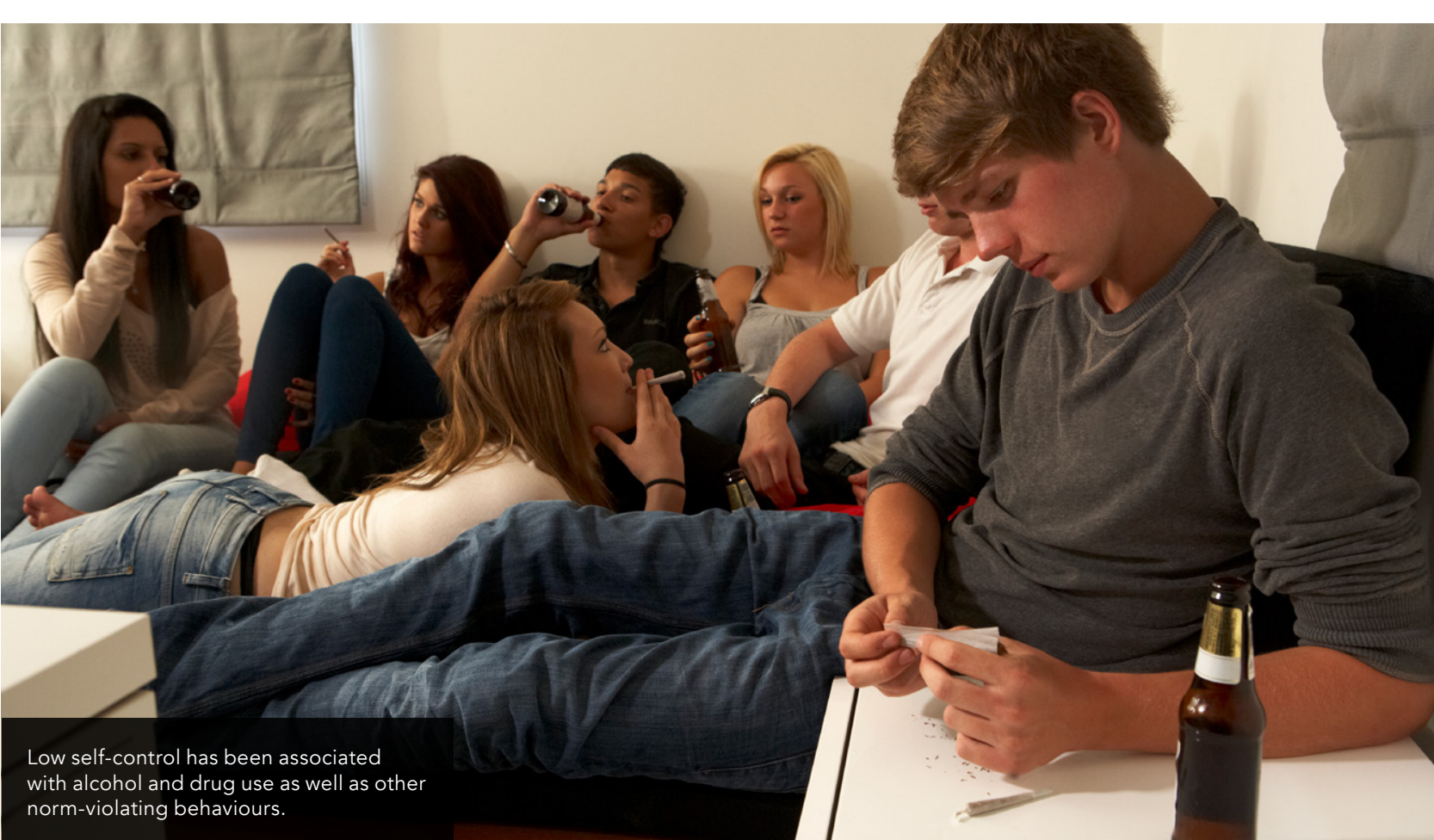

He is therefore conducting research in

They showed substantial support for low a number of countries. Prof Vazsonyi's self-control being a factor in deviance.

The researchers employed statistical techniques knomployed statistica recent work has also focused on sleep, and particularly how sleep is interw with other factors to influence adolescent behaviour. An
important pape from his group has recently delved deeper into the relationships between sleep and control.

Self-control is part of what is known as 'executive function' - a crucial part of cognition that includes planning, attention and the ability to switch between tasks. Several studies have linked poor sleep to poor executive functioning, including low self-control.

Low self-control has been associated with a number of norm-violating behaviours, including young age alcohol and drug use, delinquency, and deviance. In a recent meta-analysis, Prof Vazsonyi an colleagues looked at studies that had examined the relationship between self-control and deviance between 2000 and 2010, using statistical techniques to explore correlations in both crosssectional (i.e. looking at data at one specific point in time) and long-term over a longer period of time) studies.

Sleep is known to be related to 'norm-violating' or deviant behaviours investigate the relationships betwe both measured variables and latent, rulles of how society works. $\quad \begin{aligned} & \text { that the effect of } \\ & \text { sleep problems } \\ & \text { on delinquency }\end{aligned}$ rulles of how society works. $\quad \begin{aligned} & \text { that the effect of } \\ & \text { sleep problems } \\ & \text { on delinquency }\end{aligned}$ theory' first put forward by criminologists was indeed moderated in part by Travis Hirschi and Michael Gottfredson in 1990 to explain crime. The analysis of a decade's worth of studies showed between low self-control and deviance or crime across studies with different assessments, designs, measures of deviance and across cultures and demographic groups.

SLEEP AND ITS RELATIONSHIP WITH DEVIANCE AND LOW SELF-CONTROL

In a recent paper in the Journal of Adolescence, Prof Vazsonyi and colleagues set out to understand more about how sleep contributes to the link between self-control and devian behaviours. They did this by looking at almost 6,900 high school students in Switserland, considening self-contro across a variety of immigration and socio. predicted lower self-control and in turn and socioeconomic aroups. This is important step in highlighting ways that young people can be influenced, and indeed helped, by sleep.

\section{CULTURAL EFFECTS}

This study was particularly important because it gathered data from a large sample of teenagers - with the support of the Swiss Federal Office of Public population. It revealed differences in sleep, with the Swiss native young people (Swiss-born and Swiss parents) reporting better sleep than young people from first-and second-generation immigrant families, highlighting that in sleep sumiles are most in need of sleep support. Studies examining 


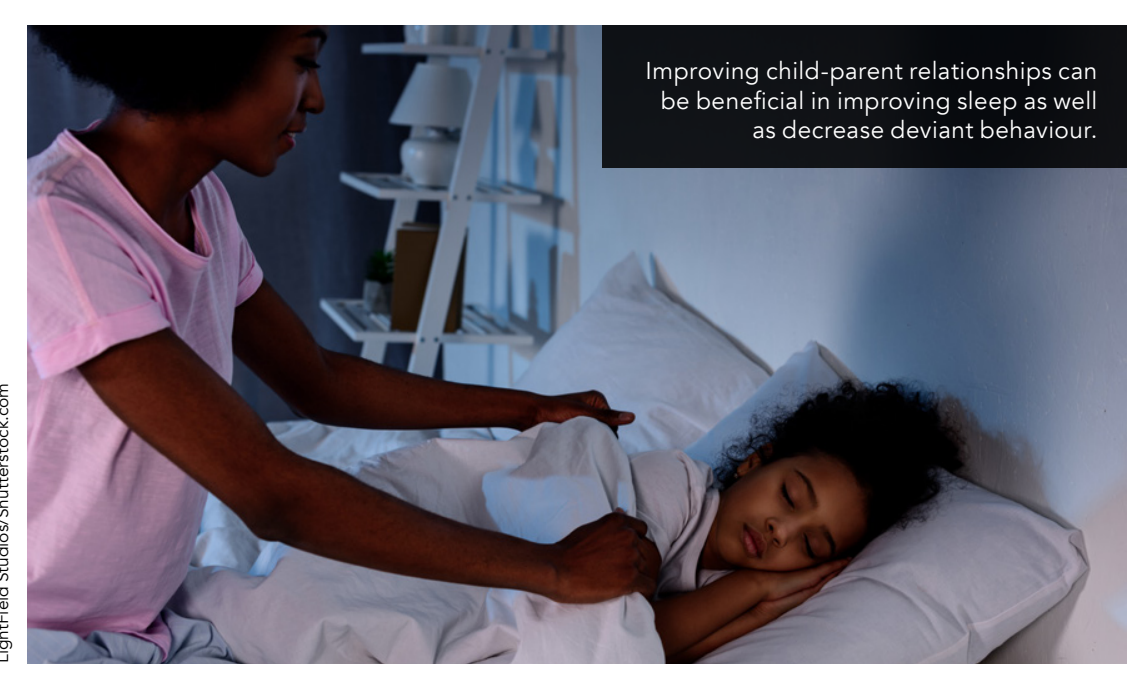

Sleep problems predicted lower selfcontrol and in turn greater delinquency across cultural and socioeconomic groups. very scarce; however, addressing this Sleep was found to mediate the effect of parental warmth on problem behaviours, stablishing the imporanghighting sleep as a mechanism that $\begin{array}{ll} & \\ \text { of American-based theories across } & \text { They found a relationship between }\end{array}$ positive parenting - measured as warm - and sleep in both countries. This is consistent with findings from previous studies stating that parental influences can improve adolescents' sleep and that young people living in homes with high monitoring have more regular sleep patterns and better-quality sleep.

IMPLICATIONS FOR INTERVENTION The Journal of Adolescence study of low self-control has several important implications. In noting that poor sleep deviance, a reasonable inference is tha tackling one of these areas could have positive outcomes on the other Primary prevention efforts to help support adolescents avoid deviance by tack and Switzerland.

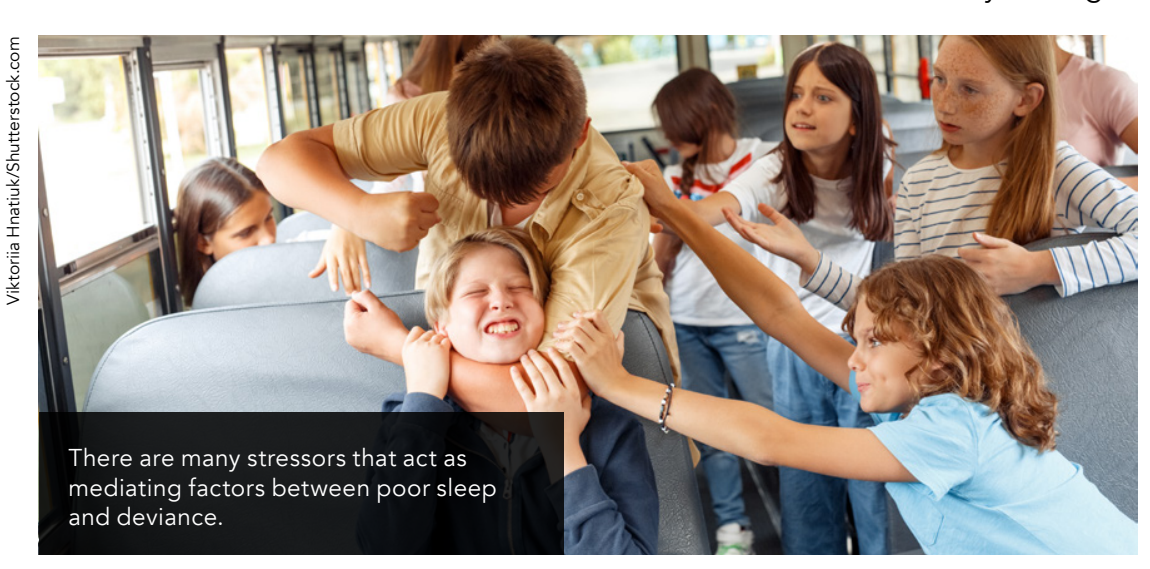

in self-control, which has been shown to be malleable, could be the way forward. Better sleep could be encouraged by better sleep hygiene. Sleep hygiene around sleeping - for example, no brigh lights in the bedroom around sleep times, maintaining stricter bedtimes and limiting screen use late in the day. Interventions that could improve sleep might reasonably be expected to reduce norm-violating behaviours and improve self-control.

The findings also suggest that improving child-parent relationships could also be beneficial, both in improving sleep and decreasing devant behaviour. Lkewise, better-quality sleep could improve family however ore peope more slep issues, these groups may be in greatest need for interventions to improve their sleep. Previous work by Prof Vazsonyi has shown that first and second-generation Swiss immigrant children are at higher risk of internalising conditions including depression. Since mental health conditions link with sleep too, this could also be an area that is improved with better sleep in young people.

\section{WHY WE CAN'T SLEEP ON IT} The work by Prof Vazsonyi and colleagues issues with widesprea sleep is an for techar we low concertration or poor school performance. Their findings not only show the relationships but also pinpoint the mediating factors (variables that link poor sleep and deviance, such as poor family relationships or other stressors) that could be part-responsible for the effects. of poor sleep on deviance. Importantly, these are areas that could be tackled in interventions. Teenagers across the world have a paucity of sleep and a wealth of sleep issues. This is particularly true of immigrant young people who are most at risk of poor sleep. Helping families to support their young people to sleep better should be a priority to affect alange not only for adolescent health but also to reduce societal problems such as demographic groups.

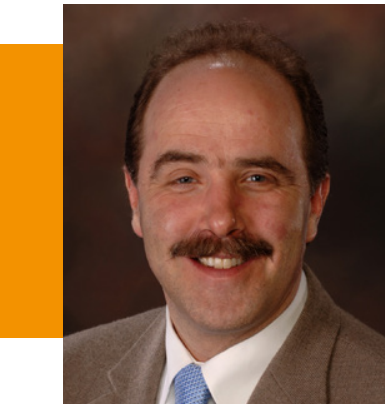

\section{Behind the Research}

\section{Dr Alexander T. Vazsonyi}

E: vazsonyi@uky.edu T: $+18592579762 \quad$ W: https://hes.ca.uky.edu/person/alexander-vazsonyi Research Objectives

Prof Vazsonyi examines the links between socioeconomic status and sleep functioning in Swiss adolescents.

\section{Detail}

Alexander T. Vazsonyi

University of Kentucky

Lexington, KY 40506-0054 (USA)

Alexander T. Vazsonyi is Professor

of Family Sciences, Professor of

at the University of Kentucky. He is

\section{References}

Vazsonyi, A.T., Ksinan, G.J., and Ksinan, A. (2018) Sleep, low self-control, and delinquency: Direct and indirect links across immigrant groups and socioeconomic strata. Journal of Adolescence, 68, 40-49. Available at: https://doi.org/10.1016/j. adolescence.2018.06.002

- Vazsonyi, A.T., Mikuska, J., and Kelley, E. (2017). It's time: A meta- analysis of the self-control-deviance link. Journal of Criminal Justice, 48, 48-63. Avallable at: https://www.doi.org//0.1016/1.jcrimjus.2016.10.001 (2016) An.T, Jiskrova, G. K., and Ksinan, A. Roma adolescents. Journal of Criminal Justice 66-76. Available at: https://www doi org/10.1016/. jcrimjus. 2015.12.004 K. Phagava, H., and Michaud, P.A.A., (2015) Para, mediation effects by sleep on the parental warmthproblem behavior links: Evidence from national probability samples of Georgian and Swiss adolescents. Journal of Youth and Adolescence, 44(2), 331-345. - Vazsonyi, A.T., Trejos, E., and Huang, L. (2006). Are developmental processes affected by immigration? Family processes, internalizing behaviors, and externalizing bes, iviors. Journal of Youth and Adolescence, 35, 795-809.

University of Kentucky。
Health (contract 00.001721/2.24.02. 81 to PA Michaud, Professor Emeritus, and with the financial support of participating cantons.

Collaborators

Prof Vazsonyi's 2 PhD students at the Gabriela Ksinan Jiskrova
What are the underlying reasons for the difference in sleep quality between adolescents of Swiss-born and immigrant parents?

This is a challenging question that likely has
many potential answers. It is possible that children and adolescents of immigrant parents experience systematically different amounts and levels of daily ranging from where they reside in a community to the typ, of school they attend. This includes attending schools with disproportionately higher levels of immigrant youth, impacting educational experiences in the classroom due to language comprehension problems as well as teacher effectiveness in delivering subject content. High amounts are known to impact sleep and vice versa.

How, in your opinion, might families best be helped? Families can be best helped by providing parents with
key information about the importance of sleep and how to best support healthy sleeping in their children and adolescents. This might include specific suggestions about at home related to the use of electronic devices that are particularly likely to interfere with youth maintaining and reaching adequate sleep behaviour goals. In addition, both teenagers and adolescents can be better educated in a playful and informative manner, of why sleep is inportant and what behaviours most inter fere with sleep 